\title{
Sample Size Adjustment
}

National Cancer Institute

\section{Source}

National Cancer Institute. Sample Size Adjustment. NCI Thesaurus. Code C142677.

A change to a previously determined sample population size for a study, following a review of blinded information during an intermediate point in the study. 